\section{Hyperfluorescence of the optic disc with indocyanine green angiography}

\author{
K Maaijwee ${ }^{1}$, PR van den Biesen ${ }^{2}$ and \\ JC van Meurs ${ }^{1,3}$
}

\begin{abstract}
Purpose One-fourth of the patients with exudative age-related macular degeneration (AMD) treated with an autologous retinal pigment epithelium (RPE)-choroid translocation had a hyperfluorescent optic disc with indocyanine green angiography (ICGA). This study aimed to identify whether indocyanine green (ICG)-assisted surgery was related to the hyperfluorescence of the optic disc with ICGA. Methods Retrospective observational case series of 31 AMD patients treated with an RPE-choroid translocation and who had ICGA after surgery. The ICGAs were assessed for hypo/iso/hyperfluorescence of the optic disc and fluorescence was related to the time interval between ICGA and the possible use of intravitreal ICG.

Results The optic disc was hyperfluorescent in six patients, isofluorescent in one, and hypofluorescent in 24 patients. All hyperfluorescent optic discs and 7 of the 24 hypofluorescent optic discs were preceded with ICG-assisted surgery with a time interval of $7 \pm 3$ weeks and $43 \pm 12$ weeks, respectively $(P=0.001$, Student $t$-test). The other 17 hypofluorescent discs were not preceded by ICG-assisted surgery and the one isofluorescent optic disc was

(ICGA) is that the optic disc is hypofluorescent in a normal angiogram. While evaluating postoperative angiograms of patients with exudative age-related macular degeneration (AMD) treated with an autologous retinal pigment epithelium (RPE)-choroid translocation, we encountered hyperfluorescent optic discs in one-fourth of these patients (Figure 1). We initially thought of an inflammatory cause, but this was highly unlikely as clinical signs for inflammation were absent, and the time interval between surgery and ICGA was too extended in most of the patients to be a plausible cause for postoperative inflammation. Another explanation could be the prolonged staining of the optic disc after intravitreal indocyanine green (ICG) administration during posterior segment surgery to visualize the inner limiting membrane (ILM). Weinberger et $a l^{1}$ first reported this in 2001. The diffusion of ICG in the optic tract was subsequently described and histological confirmed by Paques et $a l^{2}$ in an animal model.

This report aimed to identify whether ICG-assisted surgery was related to the hyperfluorescence of the optic disc in our patients and to study its persistence.
\end{abstract} observed 32 weeks after ICG-assisted surgery. Conclusion There was a statistically significant correlation between intravitreal ICG use during surgery and a hyperfluorescent optic disc with ICGA in our patient group.

Eye (2009) 23, 819-821; doi:10.1038/eye.2008.146; published online 6 June 2008

Keywords: hyperfluorescence; ICG; indocyanine green angiography; macular surgery; optic disc

\section{Introduction}

One of the first principles one learns when interpreting indocyanine green angiography

\section{Patients and methods}

Thirty-one patients with exudative AMD treated with an RPE-choroid graft translocation and who had ICGA after surgery to exclude a possible recurrent neovascular membrane, were included in this retrospective observational case series. ICGA was performed according to a routine standard protocol in all patients.

In this surgery, initial surgery with the autologous RPE-choroid graft translocation (surgery was performed as previously described by van Meurs and van den Biesen ${ }^{3}$ ) is followed by silicone oil removal after
${ }^{1}$ The Rotterdam Eye Hospital, Rotterdam, The Netherlands

${ }^{2}$ Department of Ophthalmology, University Medical Center Utrecht, Utrecht, The Netherlands

${ }^{3}$ Erasmus University, Rotterdam, The Netherlands

Correspondence: K Maaijwee, The Rotterdam Eye Hospital, Schiedamse Vest 180, Rotterdam 3011 $\mathrm{BH}$, The Netherlands.

Tel: + +31 10 4017777; Fax: + 31104111747 .

E-mail: kmaaijwee@ hotmail.com

Received: 26 November 2007

Accepted in revised form: 15 April 2008 Published online: 6 June 2008

The authors have no commercial or financial interest in this publication

Financial support: the SWOO-Flieringa Foundation (Foundation Scientific Research Eye Hospital Rotterdam), the Rotterdam Eye Hospital, Rotterdam, The Netherlands 


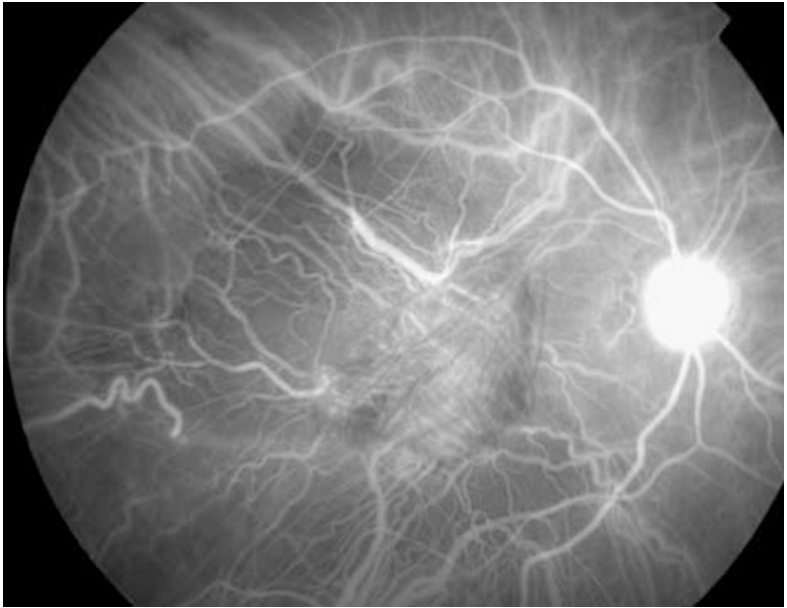

Figure 1 Indocyanine green angiography (ICGA) in patient with exudative age-related macular degeneration treated with an autologous retinal pigment epithelium-choroid translocation. ICGA shows hyperfluorescent optic disc at 3 months after intravitreal indocyanine green-assisted surgery.

Table 1 Appearance of the optic disc in indocyanine green angiography (ICGA) related to indocyanine green (ICG)-assisted surgery in 31 patients with exudative age-related macular degeneration treated with a retinal pigment epithelium-choroid translocation

\begin{tabular}{lll}
\hline $\begin{array}{l}\text { ICG-assisted } \\
\text { surgery before } \\
\text { ICGA }\end{array}$ & $\begin{array}{l}\text { Optic disc } \\
\text { staining with } \\
\text { ICGA }\end{array}$ & $\begin{array}{l}\text { Time (weeks) } \\
\text { between ICGA and } \\
\text { ICG-assisted surgery }\end{array}$ \\
\hline No $(n=18)$ & $\begin{array}{l}\text { hypofluorescent } \\
(n=18) \\
\text { Yes }(n=13)\end{array}$ & $\begin{array}{l}\text { Nyperfluorescent } \\
(n=6) \\
\text { isofluorescent } \\
(n=1)\end{array}$ \\
& $\begin{array}{l}\text { hypofluorescent } \\
(n=6)\end{array}$ & $73 \pm 3$ (range: $4-12)$ \\
\hline
\end{tabular}

3-4 months, at which time ICG-assisted ILM removal is performed in selected patients. In two patients, the ICGAs were obtained before and in 29 patients, after silicone oil removal. The ICGAs were assessed for hypo/ iso/hyperfluorescence of the optic disc and related to the time interval between ICGA and the possible use of intravitreal ICG.

\section{Results}

The optic disc was hyperfluorescent in six patients, isofluorescent in one, and hypofluorescent in 24 patients (Table 1). All ICGAs with hyperfluorescent and 7 of the 24 hypofluorescent optic discs were preceded with ICG-assisted surgery with a time interval of $7 \pm 3$ weeks and $43 \pm 12$ weeks, respectively ( $P=0.001$, Student $t$-test). The other 17 hypofluorescent discs were not preceded by
ICG-assisted surgery and the one isofluorescent optic disc was observed 32 weeks after ICG-assisted ILM peeling (Table 1).

Of the six patients with hyperfluorescent optic discs, the optic discs of both the fellow eyes (available in three patients) and operated eyes before surgery (available in five patients) were hypofluorescent.

\section{Discussion}

A causal connection between intravitreal ICG use during surgery and a hyperfluorescent optic disc with ICGA in our patient group was suggested by the statistically significant correlation and the documented neurophilic staining properties of ICG. ${ }^{2}$

Hyperfluorescence could be observed at least the first 4 months after surgery and subsequently faded away till the normal hypofluorescence was returned after about 10 months.

There are only a few articles reporting on the phenomenon of a hyperfluorescent optic disc after intravitreal ICG application and all but one have a short-term follow-up. Shortly after ICG application, hyperfluorescence of distinct areas of the retina was observed, which progressively migrated towards the optic disc with finally only the optic disc being hyperfluorescent. In these studies, hyperfluorescence of the optic disc also started to fade after about 3-6 months after surgery but could persist up to 6-12 months. ${ }^{4-7}$ Only one study recently reported on the long-term follow-up (16-36 months after surgery) in 18 patients and their results suggested that ICG might even persist for years. ${ }^{8}$ An explanation for this is that they used a high-contrast sensitivity scanning laser ophthalmoscope (HRA), which is more sensitive than ICG angiography with a conventional fundus camera used in this and several other studies, because there is a lower level of light scatter. ${ }^{8,9}$

In conclusion, since the introduction of intravitreal ICG-assisted surgery, a hyperfluorescent optic disc with ICGA may be observed more often in daily practice, a phenomenon we found to be unknown even under experienced medical retinal specialists. Therefore, if doctors see a hyperfluorescent disc in someone who recently had vitreoretinal surgery, they should ask the surgeon whether intravitreal ICG was used, rather than immediately starting a prolonged series of expensive investigations to identify an inflammatory cause.

\section{References}

1 Weinberger AW, Kirchhof B, Mazinani BE, Schrage NF. Persistent indocyanine green (ICG) fluorescence 6 weeks after ICG administration for macular hole 
surgery. Graefes Arch Clin Exp Ophthalmol 2001; 239: 388-390.

2 Paques M, Genevois O, Régnier A, Tadayoni R, Sercombe R, Gaudric A et al. Axon-tracing properties of indocyanine green. Arch Ophthalmol 2003; 121: 367-370.

3 Van Meurs JC, van den Biesen PR. Autologous retinal pigment epithelium and choroid translocation in patients with exudative age-related macular degeneration: short-term follow-up. Am J Ophthalmol 2003; 136: 688-695.

4 Kroemer I, Lommatzsch A, Pauleikhoff D. Retinal ICG accumulation after ILM staining during macular hole surgery? Ophthalmologe 2004; 101: 604-607.

5 Tadayoni R, Paques M, Girmens JF, Massin P, Gaudric A. Persistence of fundus fluorescence after use of indocyanine green for macular surgery. Ophthalmol 2003; 110: 604-608.
6 Sayanagi K, Ikuno Y, Soga K, Sawa M, Oshima Y, Kamei $\mathrm{M}$ et al. Residual indocyanine green fluorescence pattern after vitrectomy for idiopathic macular hole with internal limiting membrane peeling. Br J Ophthalmol 2007; 91: 939-944.

7 Machida S, Fujiwara T, Gotoh T. Observation of the ocular fundud by an infra-red-sensitive video camera after vitreoretinal surgery assisted by indocyanine green. Retina 2003; 23: 183-191.

8 Sekiryu T, lida T. Long-term observation of fundus infrared fluorescence after indocyanine green-assisted vitrectomy. Retina 2007; 27: 190-197.

9 Flower RW, Csaky KG, Murphy RP. Disparity between fundus camera and scanning laser ophthalmoscope indocyanine green imaging of retinal pigment epithelium detachments. Retina 1998; 18: 260-268. 